\title{
CDCA7L promotes hepatocellular carcinoma progression by regulating the cell cycle
}

\author{
YUAN TIAN $^{1 *}$, CHANGJUN HUANG $^{1 *}$, HAI ZHANG $^{1 *}$, QINGFENG NI $^{1}$, SHENG HAN $^{1}$, \\ DONG WANG $^{1}$, ZEGUANG HAN ${ }^{2}$ and XIANGCHENG LI ${ }^{1}$
}

${ }^{1}$ Liver Transplantation Center, First Affiliated Hospital of Nanjing Medical University, Key Laboratory of Living Donor Liver Transplantation, Ministry of Public Health, Nanjing; ${ }^{2}$ Shanghai-MOST Key Laboratory for Disease and Health Genomics, Chinese National Human Genome Center at Shanghai, Shanghai, P.R. China

Received August 6, 2013; Accepted September 30, 2013

DOI: 10.3892/ijo.2013.2142

\begin{abstract}
The cell division cycle-associated 7-like protein (CDCA7L) is a recently-identified target gene of c-Myc which can also interact with c-Myc. It is known to be upregulated in many tumors, however, its role in tumor progression remains unclear. We investigated the role of CDCA7L expression in hepatocellular carcinoma (HCC). We confirmed that CDCA7L is strongly upregulated in human $\mathrm{HCC}$, and demonstrated that ectopic overexpression of CDCA7L promotes HCC cell proliferation and colony formation. Conversely, knockdown of CDCA7L inhibits these malignant phenotypes. In an in vivo model, subcutaneous transplantation of the tumor in nude mice showed that overexpression of CDCA7L can accelerate the tumor growth rate. Mechanistic analyses indicated that CDCA7L was able to activate the extracellular signalregulated kinase $1 / 2(\mathrm{ERK} 1 / 2)$ signaling pathway and regulate the cell cycle, thus promoting HCC progression. Collectively, these findings show that CDCA7L plays a role in promoting the development of $\mathrm{HCC}$ and may constitute a potential therapeutic target in HCC.
\end{abstract}

\section{Introduction}

Hepatocellular carcinoma (HCC) is one of the most common malignancies worldwide, with a 5-year survival rate of only $5 \%(1-5)$. As such it is a major health problem with increasing number of new cases diagnosed every year. The major risk factors for HCC development are largely known (hepatitis B

Correspondence to: Dr Xiangcheng Li, First Affiliated Hospital of Nanjing Medical University, 300 Guangzhou Road, Nanjing 210029, P.R. China

E-mail:drxcli@njmu.edu.cn

${ }^{*}$ Contributed equally

Key words: cell division cycle-associated 7-like, hepatocellular carcinoma, cell proliferation, cell cycle, extracellular signal-regulated kinase $1 / 2$ virus, hepatitis $\mathrm{C}$ virus, alcohol, toxic chemicals), but the specific pathogenesis and progression mechanism is still not well understood (6-8).

c-Myc oncogene activation is a critical event in the pathogenesis of a large number of human malignancies, including HCC, the most common solid tumor (8-12). A number of c-Myc target genes have been identified, including CAD, ODC, CDC25A, LDH-A and cyclin E (13-19). It has previously been shown that c-Myc upregulates CDCA7L mRNA expression and colocalizes and interacts with CDCA7L. Furthermore, CDCA7L is able to complement the c-Myc transformation-defective mutant W135E and potentiate Myc-mediated transformation $(20,21)$. It has also been confirmed that CDCA7L induces colony formation, and contributes to Myc-mediated transformation of medulloblastoma cells, indicating that CDCA7L plays an important role in medulloblastoma tumor development (21). There is ample evidence indicating that CDCA7L can suppress monoamine oxidase A (MAO A) by interacting with the MAO A promoter and inhibiting enzymatic activities, this effect leads to increased expression of the cell cycle enhancers E2F1 and cyclin D, demonstrating that both CDCA7L and MAO A are involved in cell growth and apoptotic signaling pathways (20-23).

Despite many previous studies that have been carried out in this area, CDCA7L expression in HCC is still not completely known. Therefore, our objective was to examine the role of CDCA7L in HCC development. Our results indicate that CDCA7L is markedly upregulated in HCC and can act to promote HCC cell proliferation and colony formation both in vitro and in vivo. These data may provide further insight into HCC development and suggest that CDCA7L may constitute a potential therapeutic target in HCC.

\section{Materials and methods}

Patients. Sixty pairs of clinical specimens were obtained from patients who were diagnosed with $\mathrm{HCC}$ at the First Affiliated Hospital of Nanjing Medical University. Adjacent non-HCC tissues were taken $2 \mathrm{~cm}$ away from the primary cancer edge and were confirmed to be non-HCC tissues by pathological diagnosis. All patients gave their informed consent and the study was approved by the institutional ethics committee of Nanjing Medical University. 
Cell lines and cell culture. All HCC and normal human liver cell lines (Huh-7, SK-hep-1, MHCC-97H, MHCC-97L, LM3, LM6, Hep-3B, Hep-G2, YY-8103, Focus, WRL-68, L02) used in this study were obtained from the Chinese National Human Genome Center at Shanghai. All cell lines were cultured in a $5 \% \mathrm{CO}_{2}, 37^{\circ} \mathrm{C}$-humidified incubator in Dulbecco's modified Eagle's medium (DMEM; Gibco, Grand Island, NY, USA) supplemented with $10 \%$ heat-inactivated fetal bovine serum (FBS) and penicillin (50 U/ml) and streptomycin $(50 \mu \mathrm{g} / \mathrm{ml})$.

RT-PCR and real-time PCR. Total RNA was extracted using TRIzol $^{\circledR}$ (Invitrogen) according to the manufacturer's protocol. Reverse transcription (RT) was performed in a $25 \mu \mathrm{l}$ reaction mix with $2 \mu \mathrm{g}$ RNA using an M-MLV reverse transcriptase kit (Promega). The sequences of primers used in these experiments were as follows: CDCA7L-457 bp, 5'-CGACTCGCTACCAG ATCCCT-3' (forward) and 5'-TTGTTGGCCAGCTTCTT GGT-3' (reverse); CDCA7L-178 bp, 5'-TTGGCGACTCGCTA CCAGAT-3' (forward) and 5'-AATGAAAGCGCACATC CTGC-3' (reverse); $\beta$-actin-230 bp: 5'-AGAGCCTCGCCTTT GCCGATCC-3' (forward) and 5'-CTGGGCCTCGTCGCCCA CATA-3' (reverse).

Construction of the CDCA7L expression vector. The CDCA7L open reading frame (ORF) was amplified from a human liver cDNA library (GeneBank accession no.: NM_018719.4) using Prime Star PCR, and then inserted into pcDNA3.1B-Flag, obtained from the Chinese National Human Genome Centre at Shanghai. The sequences of the cloning primers were as follows: CDCA7L-EcoRI forward primer (5'-AGAGAATTC ATGGAGTTGGCGACTCGCTAC-3'), CDCA7L-BamHI reverse primer (5'-AGAGGATCCATTGTCTTCTACCAG CTCCTT-3'). The final sequence of the CDCA7L open reading frame (ORF) was confirmed by DNA sequencing.

siRNA preparation and shRNA cloning. Small interference RNAs (RNAi) were chemically synthesized (GenePharma Co.). Two siRNAs against CDCA7L were designed and their sequences were as follows: siRNA-834, 5'-GCCAGAUUUC UUCCCAGUAdTdT-3' (sense) and 5'-UACUGGGAAGAA AUCUGGCdTdT-3' (antisense); siRNA-1020, 5'-CCGAAGA AGGAAGACAAUUdTdT-3' (sense) and 5'-AAUUGUCUUC CUUCUUCGGdTdT-3' (antisense). A common sequence was used as negative control: siRNA-NC, 5'-UUCUCCGAACGUG UCACGUdTdT-3' (sense); 5'-ACGUGACACGUUCGGAGAA dTdT-3' (antisense). The oligonucleotides encoding short hairpin RNAs (shRNA) were synthesized and inserted into pSUPER vector, and designated pSUPER-shNC, pSUPER-sh834, and pSUPER-sh1020, respectively. Their sequences were: shRNA-NC, sense, 5'-GATCCCCTTCTCCGAACGTGTCAC GTTTCAAGAGAACGTGACACGTTCGGAGAATTTTTG GAAA-3'; antisense, 5'-AGCTTTTCCAAAAATTCTCGAA CGTGTCACGTTCTCTTGAAACGTGACACGTTCGGAG AAGGG-3'; shRNA-834, sense, 5'-GATCCCCGCCAGATTT CTTCCCAGTATTCAAGAGATACTGGGAAGAAATCTG GCTTTTTGGAAA-3'; antisense, 5'-AGCTTTTCCAAAAA GCCAGATTTCTTCCCAGTATCTCTTGAATACTGGGAA GAAATCTGGCGGG-3'; shRNA-1020, sense, 5'-GATCCCC CCGAAGAAGGAAGACAATTTTCAAGAGAAATTGTCT TCCTTCTTCGGTTTTTGGAAA-3'; antisense, 5'-AGCTTT
TCCAAAAACCGAAGAAGGAAGACAATTTCTCTTGAA AATTGTCTTCCTTCTTCGGGGG-3'.

Cell transfection. Cell transfection was performed using Lipofectamine 2000 (Invitrogen) according to the manufacturer's instructions. Cells were transfected with RNAi and plasmid at cell density 30-50 and 80-90\%, respectively.

Cell proliferation. For the proliferation assay, 2,000-5,000 HCC cells were plated into 96-well plates and cultured for about a week. Cell viability was measured using the Cell Counting Kit-8 (CCK-8, Dojindo Laboratories) according to the manufacturer's instructions. Absorbance was measured at $450 \mathrm{~nm}$ each day and used to plot a cell growth curve.

Colony formation assay and soft-agarose colony formation assay. HCC cells were transfected and then plated onto $10 \mathrm{~cm}$ plates, at a seeding density of 10,000-50,000 cells. Transfected cells were selected using G418 (Life Technologies Inc.) which was added to the medium at a final concentration of $0.8-1 \mathrm{mg} / \mathrm{ml}$. After 2-3 weeks, all clones were stained with Coomassie Brilliant Blue.

For the soft agar assay, 2,000-5,000 transfected cells were plated into culture dishes in complete medium mixed with $0.5 \%$ agarose over a bottom layer of complete medium mixed with $1 \%$ agarose. After culture for 2-3 weeks, all clones were photographed and counted under a microscope.

Western blot analysis. Western blot analysis was performed according to the manufacturer's instructions (Santa Cruz Biotechnology). Cell lysates were prepared in lysis buffer [25 mmol/1 Tris (pH 6.8), 1\% SDS, 5 mmol/l EDTA, protease inhibitor cocktail (Sigma)], and total protein was assayed using a BCA kit. The samples were subjected to SDS-PAGE on a $10 \%$ gel, then transferred to a polyvinylidene difluoride (PVDF) membrane. Protein blots were blocked with $5 \%$ milk and $0.1 \%$ Tween-20 in PBS for $2 \mathrm{~h}$ at room temperature, then incubated with the primary antibody at $4{ }^{\circ} \mathrm{C}$ overnight. After washing, blots were incubated with the secondary antibody at room temperature for $1 \mathrm{~h}$ and labeled protein was detected using the Odyssey Infrared Imaging System (Li-COR). Mouse antibodies to cyclin D1 and $\beta$-actin (Santa Cruz Biotechnology), goat antibody to CDCA7L (Santa Cruz Biotechnology) and rabbit antibodies to pERK1/2 and total ERK1/2 (Cell Signaling Technology) were used in this study.

In vivo subcutaneous HCC tumor model. Approximately $5 \times 10^{6} \mathrm{HCC}$ cells were injected subcutaneously into the flank of male nude mice (4-6 weeks old). Tumors were measured and weighed 3-4 weeks after injection or when the tumor mass reached $1,500 \mathrm{~mm}^{3}$. Tumor size was measured with calipers every 3 days, and tumor volume was determined using the following formula: volume $=0.5 \mathrm{x}$ width ${ }^{2} \mathrm{x}$ length .

Cell cycle analysis and cell synchronization. Flow cytometry was used to analyze the cell cycle. First, cells were cultured in serum-free medium for $24 \mathrm{~h}$ to induce cell cycle synchronization. These cells were then transfected with siRNA or plasmid and then harvested at a different time point. For DNA content analysis, cells were fixed in $70 \%$ ethanol, rehydrated in PBS, 
A

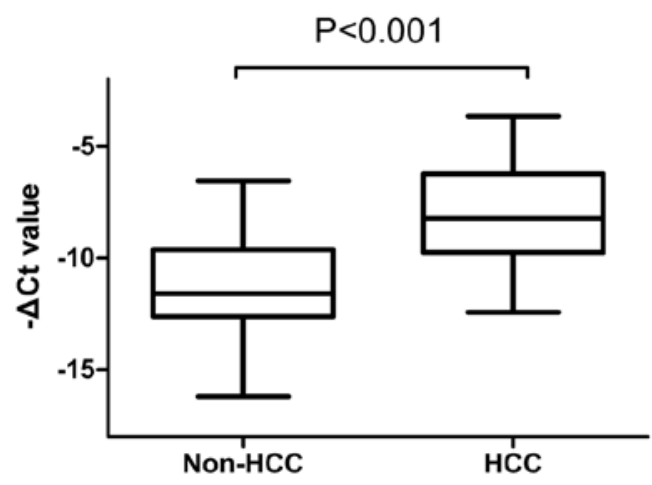

C

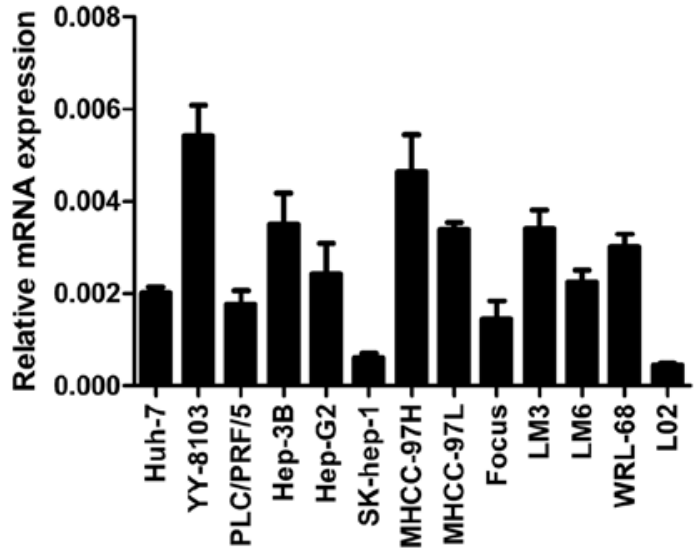

B

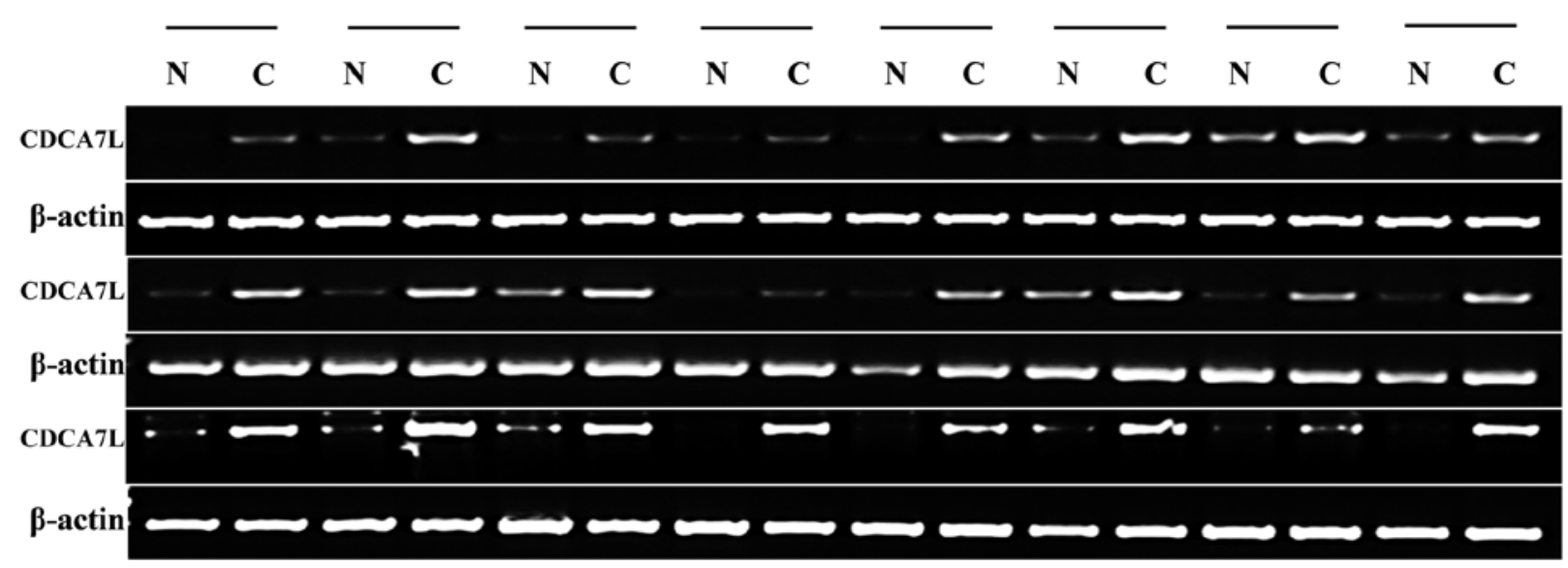

Figure 1. Expression pattern of CDCA7L in HCC specimens and cell lines. (A) The mRNA level of CDCA7L in 60 paired HCC specimens and in corresponding non-HCC tissue samples was analyzed by quantitative real-time PCR, with $\beta$-actin as internal control. The line within each box represents the median - $\Delta \mathrm{Ct}$ value, while the upper and lower bars indicate the highest and lowest values detected, respectively. The P-value was calculated by Student's t-test. (B) Representative results of agarose gel electrophoresis showing upregulation of CDCA7L in 24 paired HCC specimens by semi-quantitative RT-PCR. (C) The relative mRNA level of CDCA7L was evaluated in $12 \mathrm{HCC}$ cell lines and in the normal human liver cell line L02 by quantitative RT-PCR, with $\beta$-actin as internal control.

treated with RNase A (10 mg/ml) for 30 min then stained with propidium iodide $(10 \mu \mathrm{g} / \mathrm{ml})$ for $5 \mathrm{~min}$.

Statistical analysis. All experimental data were analyzed by Student's t-test using GraphPad Prism 5 software. In all tests, $\mathrm{P}<0.05$ was considered significant.

\section{Results}

CDCA7L is significantly upregulated in HCC. The expression level of CDCA7L was evaluated in 60 paired human HCC specimens by quantitative and semi-quantitative RT-PCR. The results showed that CDCA7L was overexpressed in 42 of $60 \mathrm{HCC}$ tissue specimens compared with the corresponding non-HCC tissue specimens (Fig. 1A and B). Furthermore, when the expression level of CDCA7L in HCC cell lines and a normal human liver cell line was analyzed by real-time PCR, the results showed that CDCA7L was highly expressed in YY-8103 and MHCC-97H HCC cell lines but low in SK-hep-1 and Focus HCC cell lines, while CDCA7L expression in the normal human liver cell line L02 was lower than in any of the HCC cell lines tested (Fig. 1C).
CDCA7L overexpression promotes HCC cell proliferation and colony formation. To determine the effect of CDCA7L expression in HCC cells, the recombinant plasmid pcDNA3.1-CDCA7L (CDCA7L) was transfected into SK-hep-1 and Focus cells, to observe and measure cellular proliferation, colony formation and soft agar colony formation. Cells transfected with empty plasmid pcDNA3.1 (vector) were used as control. The expression of exogenous CDCA7L protein was identified by western blot analysis. Analysis of cell proliferation and colony formation revealed that SK-hep-1 and Focus cells transfected with pcDNA3.1-CDCA7L (CDCA7L) had increased cell viability and produced more colonies than the corresponding cells transfected with pcDNA3.1 (vector) (Fig. 2A, B, D and E). In order to determine the effect of CDCA7L on anchorage-independent growth, a soft agar colony formation experiment was performed, and again, compared with control cultures, ectopic CDCA7L resulted in a significant enhancement of cellular colony formation by SK-hep-1 and Focus cells (Fig. 2C and F). Taken together, these data indicate that CDCA7L overexpression enhances HCC cellular proliferation, colony formation and soft agar colony formation. 
A

\section{SK-hep-1}

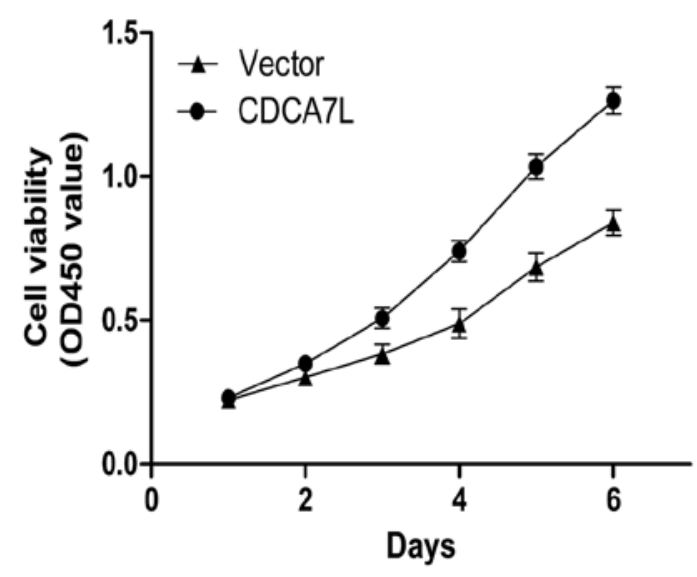

Flag

$\beta$-actin

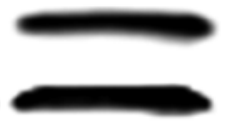

Vector

CDCA7L

B

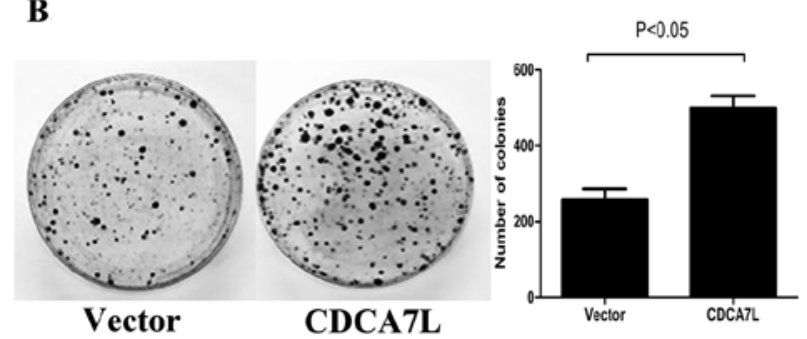

C

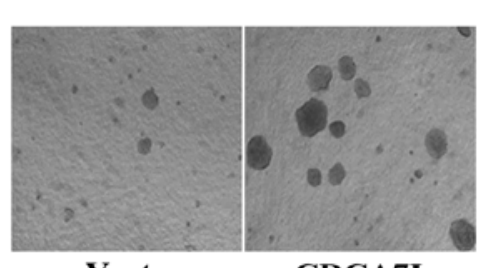

Vector
CDCA7L

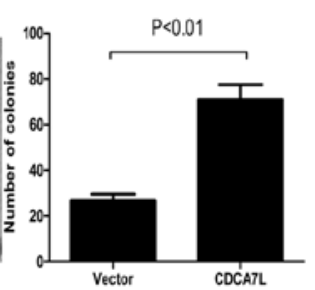

D

Focus

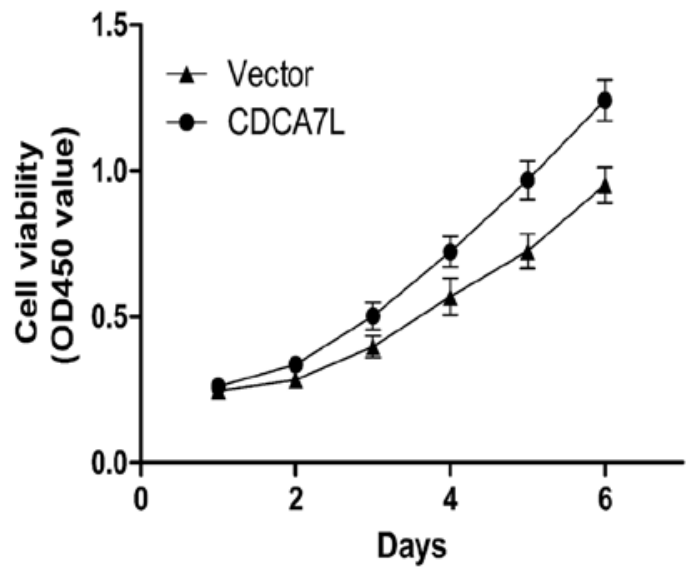

Flag

$\beta$-actin

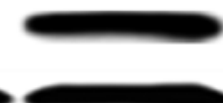

Vector

CDCA7L

E

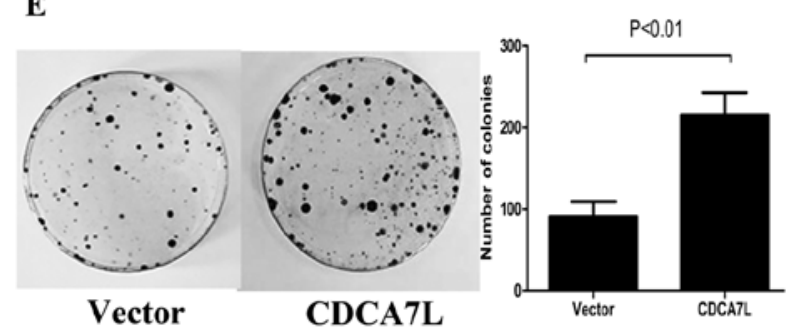

F
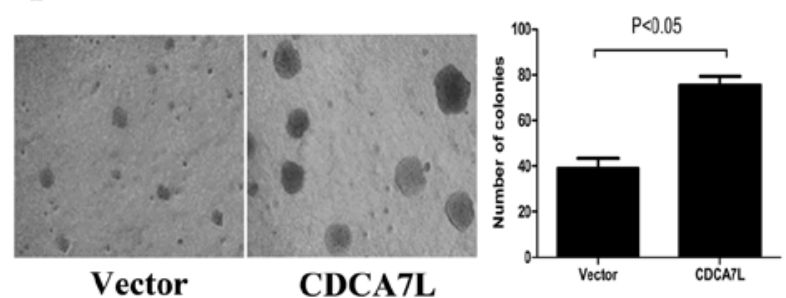

Figure 2. CDCA7L overexpression promotes HCC cell proliferation, colony formation and soft agar colony formation. (A and D) Ectopic CDCA7L expression promoted cellular proliferation of SK-hep-1 and Focus cells compared with vector (pcDNA3.1) control. The different symbols represent the corresponding mean values of the experiments, performed in triplicate (mean $\pm \mathrm{SD}$ ), $\mathrm{P}<0.05$ was calculated on days 4,5 and 6 . The expression of exogenous CDCA7L was evaluated by western blot analysis using anti-Flag antibody, with $\beta$-actin used as loading control. (B and E) CDCA7L overexpression enhanced colony formation in SK-hep-1 and Focus cells compared with vector (pcDNA3.1). Representative dishes are shown, and histograms represent the mean (n=3) and SD of the numbers of colonies. (C and F) Overexpression of CDCA7L promotes colony formation by SK-hep-1 and Focus cells in soft agar. Representative photomicrographs (x40) are shown, while histograms represent the mean $(n=6)$ and SD of colony numbers.

CDCA7L knockdown inhibits HCC cell proliferation and colony formation. To further investigate the effect of CDCA7L on cell proliferation, colony formation and soft agar colony formation, we synthetized three specific siRNAs (si-NC, si834 and si1020) and constructed the corresponding shRNA plasmids (pSUPER-shNC, pSUPER-sh834 and pSUPER-sh1020) to knock down endogenous CDCA7L in selected HCC cell lines. After evaluating the efficiency of siRNA, si834 and si1020 were considered appropriate for CDCA7L knockdown, while as negative control, si-NC was not able to knock down expression of any gene. SiRNA was capable of silencing target gene expression for a short period, while pSUPER-shRNA was able to maintain this effect indefinitely. YY-8103 and MHCC-97H cells transfected with si-NC, si834 or si1020 were used for the cell proliferation experiment. The same cells transfected with pSUPER-shNC, pSUPER-sh834 or pSUPER-sh1020 were used for colony formation and soft agar colony formation experiments. The cell growth curve revealed that cell viability of YY-8103 and MHCC-97H cells transfected with si-NC was greater than that of the corresponding 
$\mathbf{A}$

YY-8103
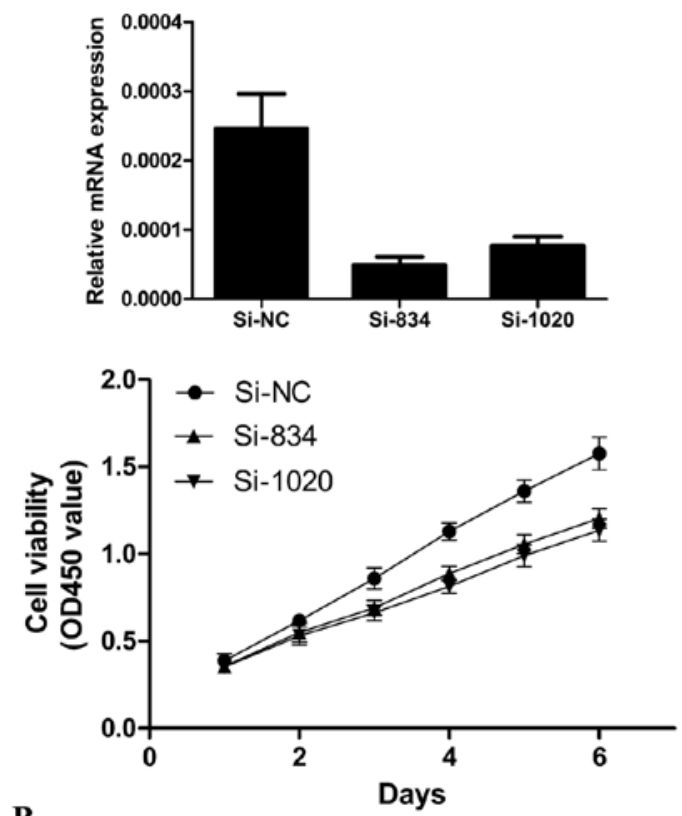

B
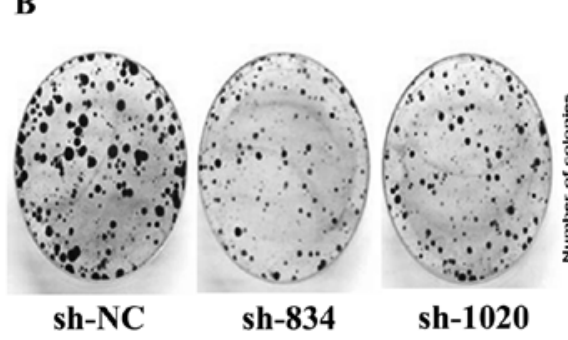

sh-1020

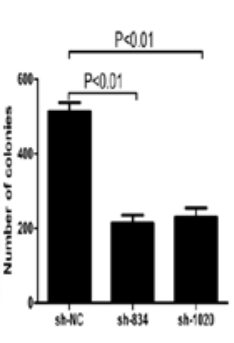

D
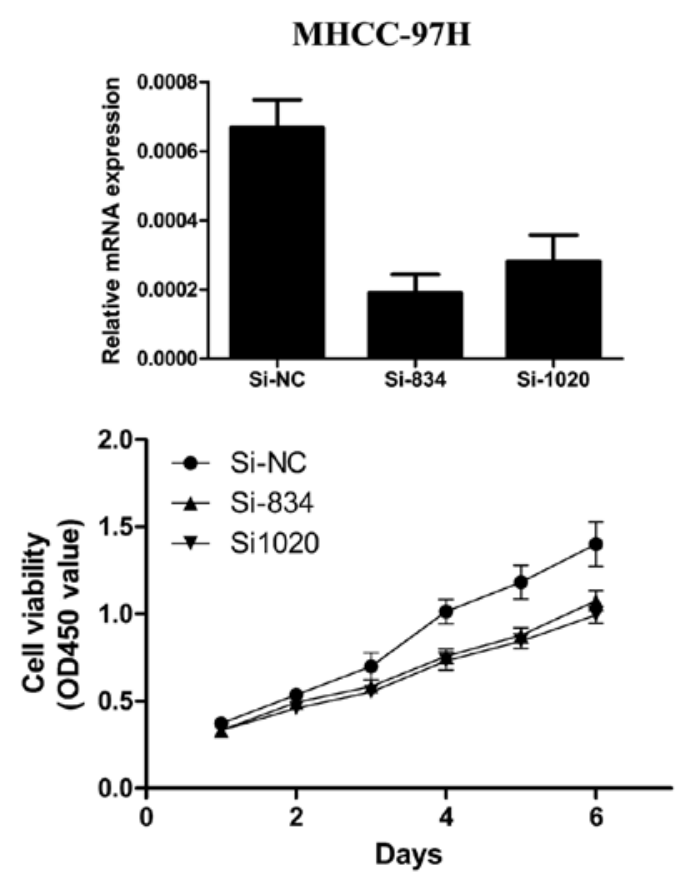

$\mathbf{E}$

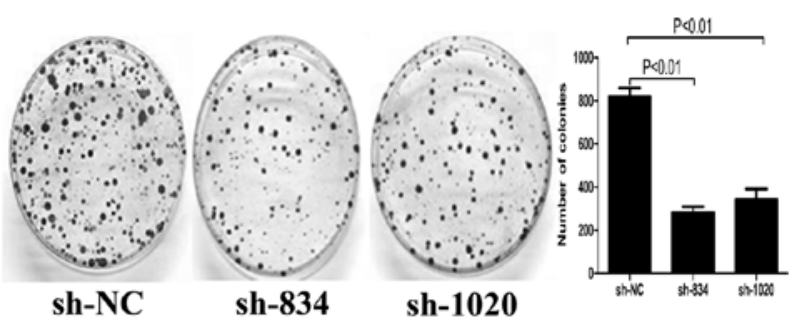

$\mathbf{F}$

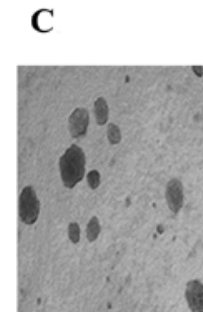

sh-NC

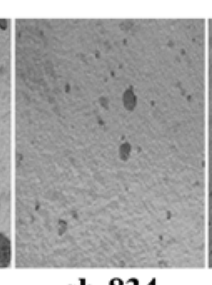

sh-834

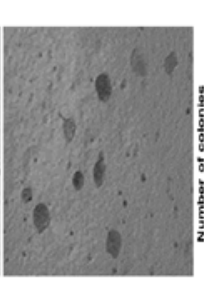

sh-1020

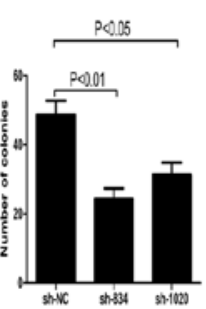

shic shes sation

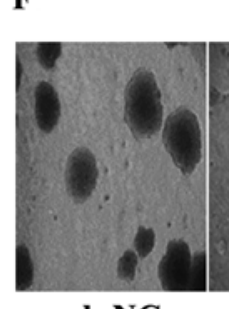

sh-NC

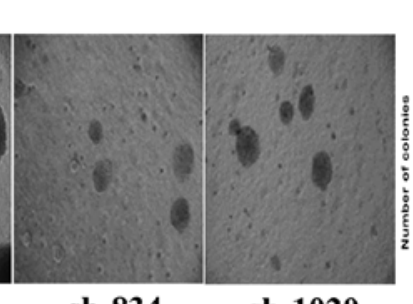

sh-834

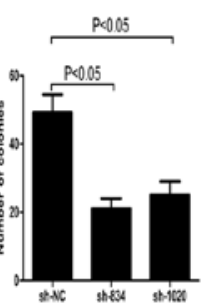

sh-1020

Figure 3. CDCA7L knockdown suppresses cell growth, colony formation and soft agar colony formation. (A and D) The knockdown efficiencies of si-834 and si-1020 in YY-8103 and MHCC-97H cells were measured by quantitative real-time PCR. Silencing CDCA7L by si-834 and si-1020 inhibited cell proliferation compared with si-NC used as control. The different symbols represent the corresponding mean values of triplicate experiments (mean $\pm \mathrm{SD}$ ), $\mathrm{P}<0.05$ was calculated on days 4, 5 and 6. Both pSUPER-sh834 and pSUPER-sh1020 inhibited (B and E) colony formation and (C and F) soft agar colony formation in YY-8103 and MHCC-97H cells compared with pSUPER-shNC-transfected controls. Photographs (x40) of areas of colony formation and representative dishes are shown; the histograms represent the mean $(n=6, n=3$, respectively) and $S D$ of the numbers of colonies.

cells transfected with si834 or si1020 (Fig. 3A and D). Likewise, YY-8103 and MHCC-97H cells transfected with pSUPER-shNC formed more, larger colonies compared with the corresponding cells transfected with pSUPER-sh834 and pSUPER-sh1020 (Fig. 3B, C, E and F). These collective data indicate that knockdown of CDCA7L can inhibit HCC cell proliferation, colony formation and soft agar colony formation.

CDCA7L overexpression contributes to tumorigenicity while CDCA7L knockdown suppresses tumorigenicity and reduces tumor burden. To further confirm the promotional effect of
CDCA7L on cell proliferation and colony formation, a subcutaneous xenograft tumor model in male nude mice was used to evaluate the effect of CDCA7L on tumorigenicity. Compared with SK-hep-1 cells transfected with empty vector plasmid pcDNA3.1 (vector), injection of the same cells transfected with pcDNA3.1-CDCA7L (CDCA7L) resulted in larger tumors in male nude mice under observation about 3 to 4 weeks after injection (Fig. 4A-C). To determine the effect of CDCA7L knockdown on tumorigenicity in vivo, pSUPER-shRNA plasmids were used to transfect YY-8103 cells, then these cells were harvested and injected into the flank of male nude mice. 
A

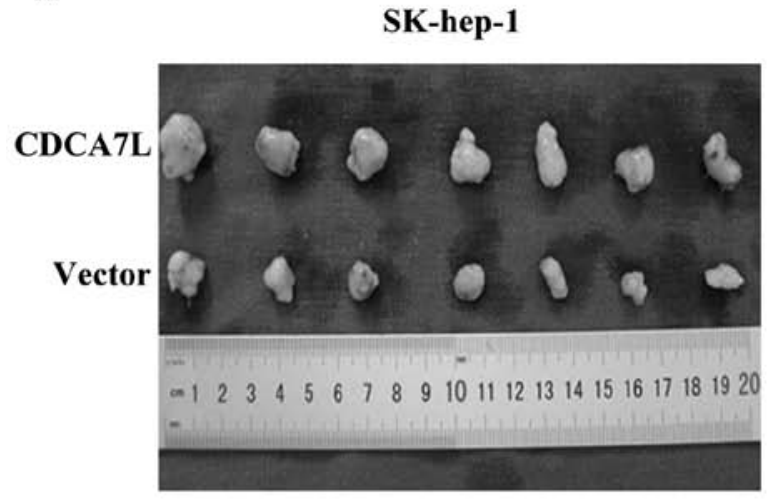

B

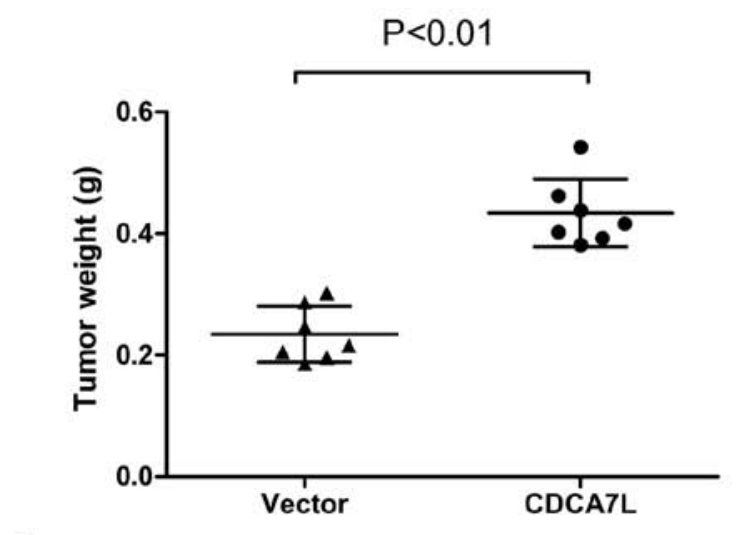

C

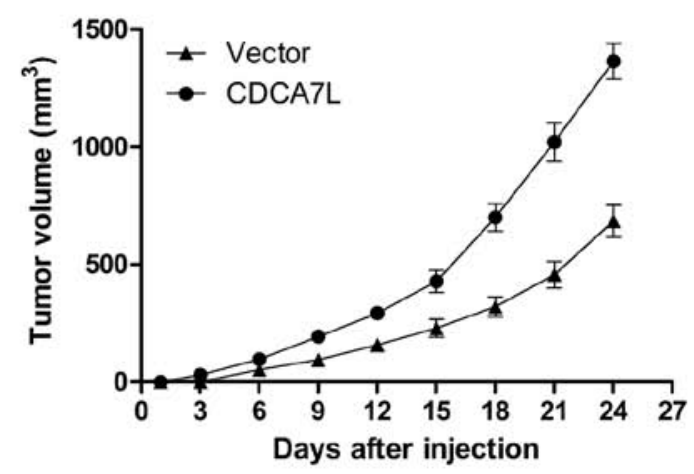

D

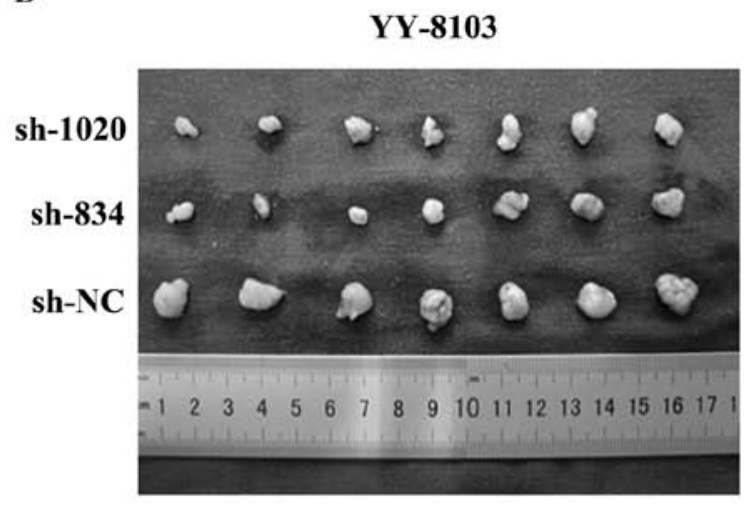

E

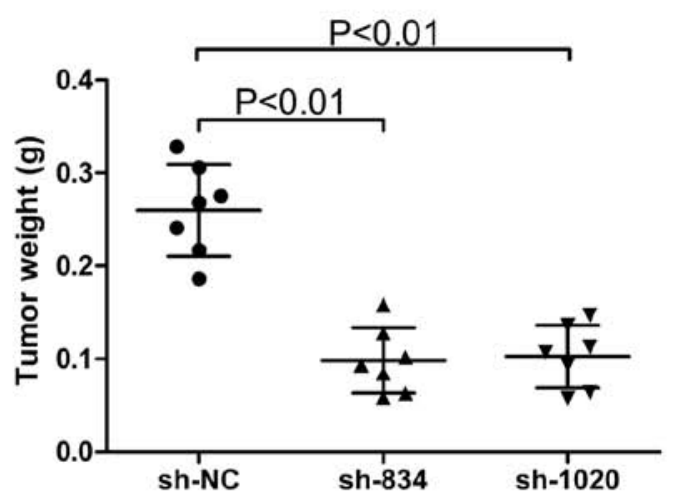

F

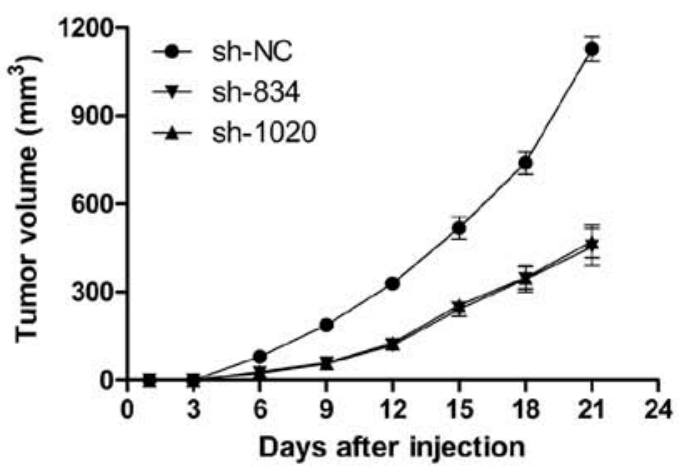

Figure 4. Overexpression of CDCA7L contributes to tumorigenicity while CDCA7L knockdown reduces the tumor burden in vivo. (A-C) Overexpression of CDCA7L enhances the tumorigenicity of SK-hep-1 cells in the flank of nude mice $(n=7)$, compared to the same cells transfected with vector (pcDNA3.1) injected into the opposite flank of the same mice as controls. Tumors were weighed (B) and tumor volume was measured every 3 days (C). (D-F) Both pSUPER-sh834 and pSUPER-sh1020 knockdown of CDCA7L suppressed the tumorigenicity of YY-8103 cells in nude mice ( $\mathrm{n}=7)$, as compared with the negative control pSUPERshNC. Tumors were weighed (E) and tumor volume was evaluated every 3 days (F).

As expected, pSUPER-sh834 and pSUPER-sh1020 were able to significantly suppress tumorigenicity, resulting in obvious reductions in tumor weight and volume compared to the negative controls pSUPER-shNC (Fig. 4D-F). All these data indicate that CDCA7L plays a role in $\mathrm{HCC}$ tumorigenicity.

CDCA7L promotes cell cycle progression. Flow cytometry was used to evaluate the effect of CDCA7L on the cell cycle. After cell cycle synchronization, these cells were transfected with plasmids or siRNA and then harvested, and their cell cycle distribution was analyzed. The results showed that enforced expression of CDCA7L caused SK-hep-1 cells to progress from G0/G1 phase and enter into $S$ phase while the same cells transfected with pcDNA3.1 (vector) did not show the same progression (Fig. 5A). Conversely, si834 and si1020 knockdown of endogenous CDCA7L in YY-8103 cells led to G0/G1 arrest while transfection with negative control si-NC had no effect (Fig. 5B). These collective data suggest that CDCA7L could contribute to HCC cell cycle progression by promoting the entry of cells from $\mathrm{G} 0 / \mathrm{G} 1$ phase into $\mathrm{S}$ phase.

CDCA7L activates the ERK1/2 signaling pathway and positively regulates cyclinDl expression. To further investigate the mechanism underlying the effect of CDCA7L on the cell cycle, 
A

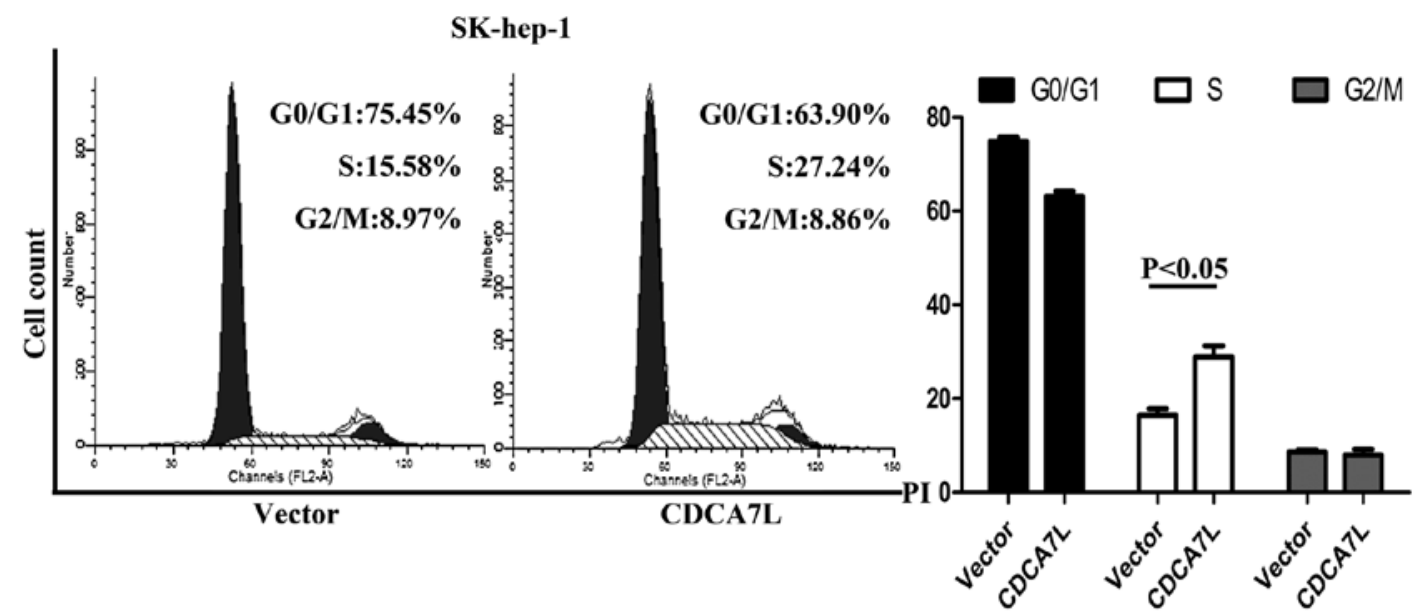

B
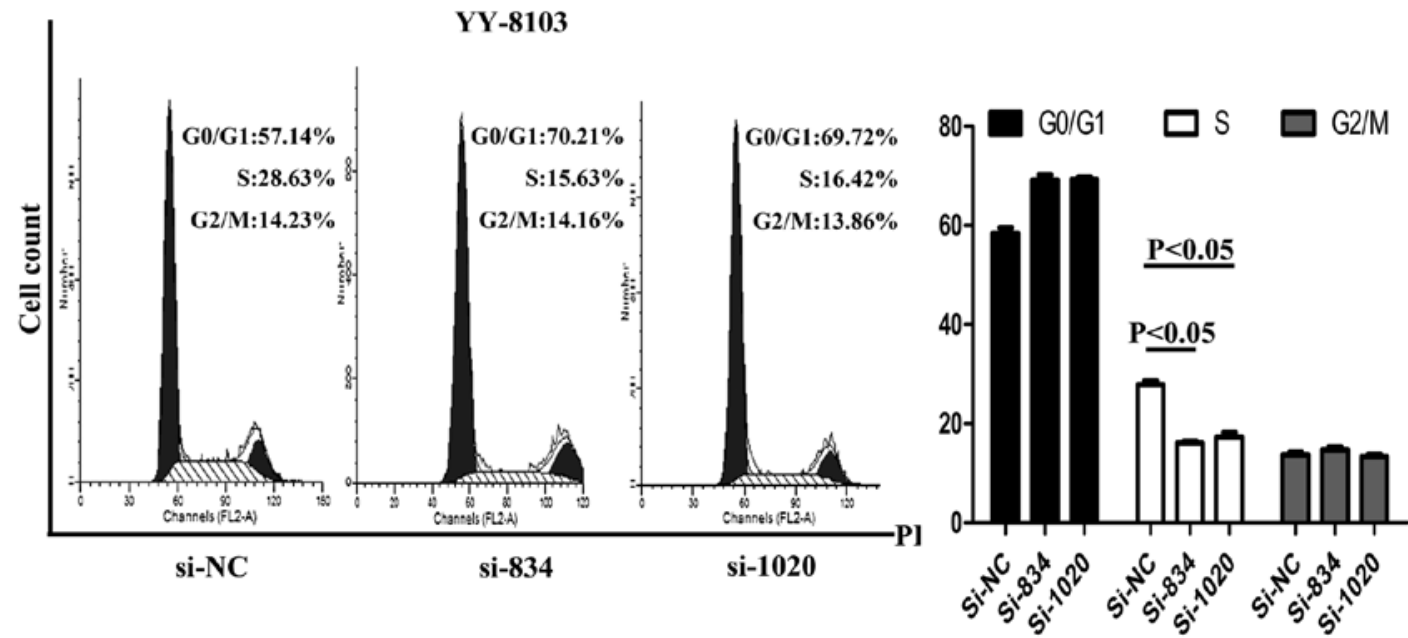

Figure 5. Overexpression of CDCA7L promotes entry of HCC cells into S phase from G0/G1 phase while knockdown of CDCA7L causes cell cycle arrest in G0/G1 phase. (A) SK-hep-1 cells were harvested $24 \mathrm{~h}$ after transfection and analyzed by flow cytometry. The histograms represent the percentages in each phase of the cell cycle, showing that ectopic CDCA7L significantly promoted the transition of G0/G1 to S phase compared with vector (pcDNA3.1) control. (B) YY-8103 cells were transfected with siRNA and harvested $24 \mathrm{~h}$ after transfection. Histograms show that knockdown of CDCA7L results in obvious arrest in G0/G1 phase compared with the negative control si-NC.

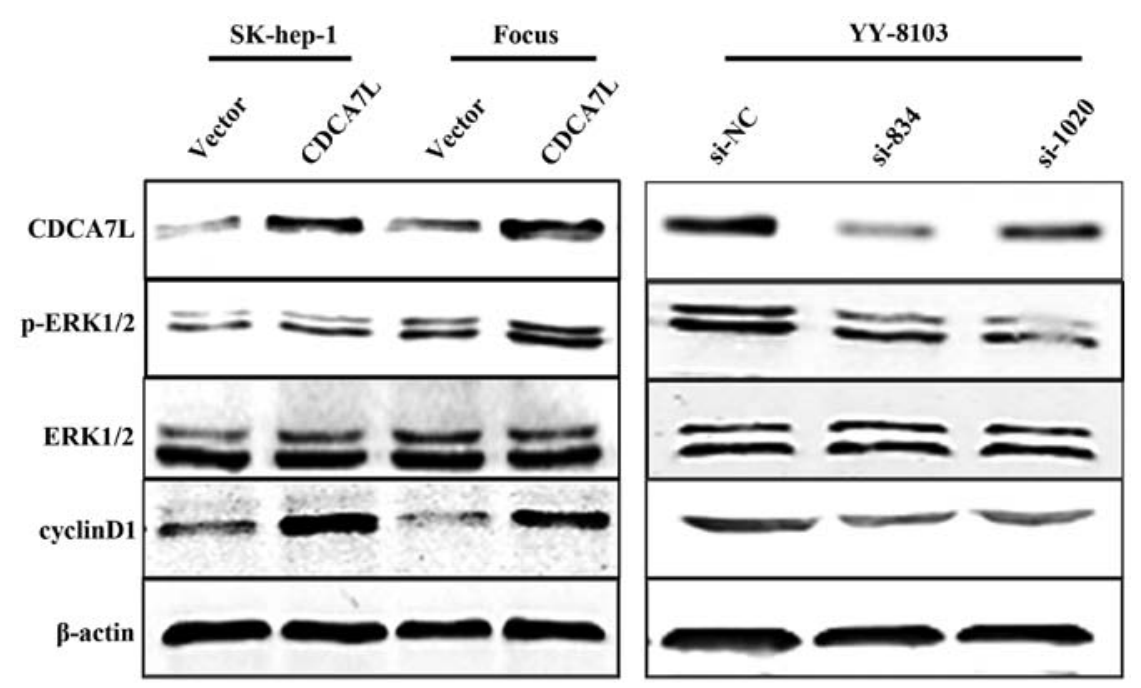

Figure 6. CDCA7L contributes to ERK1/2 activation and regulates cyclin D1 expression in a dominant-positive manner. CDCA7L overexpression significantly upregulates the levels of phosphorylated ERK1/2 and cyclin D1 proteins compared with vector (pcDNA3.1) in SK-hep-1 and Focus cells, although the level of total ERK1/2 protein remains unchanged; $\beta$-actin was used as the loading control. In YY-8103 cells, CDCA7L knockdown causes downregulation of phosphorylated ERK1/2 and cyclin D1 expression while total ERK1/2 remains unchanged. 
we first analyzed protein expression of cyclin D1 using western blot analysis. Our data showed that the cyclin D1 protein was significantly upregulated in SK-hep-1 and Focus HCC cells transfected with pcDNA3.1-CDCA7L (CDCA7L) compared with the same cells transfected with the pcDNA3.1 negative control (vector). As expected, CDCA7L knockdown caused a reduction in the level of cyclin D1 protein in YY-8103 cells compared with the negative control. It is known that activation of the ERK1/2 signaling pathway can result in upregulation of cyclin D1 expression, so we next measured the levels of phosphorylated and total ERK1/2 protein. The results revealed that phosphorylated ERK1/2 was increased in SK-hep-1 and Focus cells transfected with pcDNA3.1-CDCA7L (CDCA7L) and reduced in YY-8103 cells treated with si834 or si1020, while the level of total ERK1/2 was unchanged (Fig. 6). These data indicate that CDCA7L is involved in activation of ERK1/2 signaling and regulation of the cell cycle.

\section{Discussion}

$\mathrm{HCC}$ is the fifth most common malignancy and the third highest cause of cancer-related death worldwide, and its incidence is increasing $(24,25)$. The c-Myc oncogene is overexpressed in most cases of HCC, and is known to play an important role in cell proliferation, differentiation and apoptosis (26). c-Myc acts as a transcriptional regulator and promotes carcinogenesis by regulating a series of target genes, but the biological effects of these genes in HCC development remain unclear. CDCA7 and CDCA7L belong to the JPO protein family, recently identified as target genes of c-Myc $(27,28)$. CDCA7 expression is highly elevated during blast crises in chronic myelogenous leukemia as compared with the chronic phase, and CDCA7 transgenic mice have been shown to have a 2 -fold increase in the risk of developing lymphoid malignancies. These findings indicate that CDCA7 is involved in the development of hematologic malignancies (29). Interestingly, CDCA7L expression is upregulated, and may promote cellular proliferation, in medulloblastoma cells. Both CDCA7 and CDCA7L are capable of interacting with c-Myc and complement and potentiate Myc-mediated transformation, and could thus further participate in neoplastic transformation and contribute to tumorigenesis (27-30).

In this study, we first determined the expression of CDCA7L in HCC, and further investigated its function in HCC cell proliferation and tumorigenicity both in vitro and in vivo. CDCA7L and CDCA7 are homologous analogues; they both belong to the JPO protein family, also called the cell division cycle associated (CDCA) protein family. We therefore analyzed CDCA7L expression in HCC cells during the cell cycle and found that CDCA7L promotes entry of HCC cells into S phase from $\mathrm{G} 0 / \mathrm{G} 1$ phase. Several studies have reported that regulation of the cell cycle G1/S transition check-point is often abnormal in tumor cells (31), with the cyclins or CDK proteins and their upstream regulators changed accordingly (32-34,37). It is known that cyclin D1 is required for cell cycle progression from G0/G1 phase to $S$ phase $(35,36)$, and that sustained ERK1/2 activity can upregulate cyclin D1 expression (31), so we next determined the levels of phosphorylated and total ERK1/2 and cyclin D1 proteins using western blot analysis.

In summary, our findings show that CDCA7L is significantly upregulated in HCC, while ectopic CDCA7L expression promotes cell proliferation, colony formation, soft agar colony formation and tumorigenicity of SK-hep-1 and Focus HCC cells. Likewise, knockdown of CDCA7L inhibits cell proliferation and colony formation and reduces tumor burden of YY-8103 and MHCC-97H HCC cells. Further investigation revealed that $\mathrm{CDCA} 7 \mathrm{~L}$ stimulates ERK1/2 signaling and regulates the cell cycle by promoting the entry of HCC cells into $\mathrm{S}$ phase from G0/G1 phase. This study is the first to discover that CDCA7L expression plays an important role in $\mathrm{HCC}$ progression, but the specific role of CDCA7L in HCC remains worthy of further investigation.

\section{Acknowledgements}

This study was supported by National Natural Science Foundation, China (grant no. 81170415).

\section{References}

1. Kan Z, Zheng H, Liu X, et al: Whole genome sequencing identifies recurrent mutations in hepatocellular carcinoma. Genome Res 23: 1422-1433, 2013.

2. Wei Y, Doria C and Liu Y: Targeted therapies in the treatment of advanced hepatocellular carcinoma. Clin Med Insights Oncol 7: 87-102, 2013.

3. Dai L, Lei N, Liu M and Zhang JY: Autoantibodies to tumorassociated antigens as biomarkers in human hepatocellular carcinoma (HCC). Exp Hematol Oncol 2: 15-21, 2013.

4. Shiraha H, Yamamoto $\mathrm{K}$ and Namba $\mathrm{M}$ : Human hepatocyte carcinogenesis (Review). Int J Oncol 42: 1133-1138, 2013.

5. Wang X, Chen J, Li F, et al: MiR-214 inhibits cell growth in hepatocellular carcinoma through suppression of $\beta$-catenin. Biochem Biophys Res Commun 428: 525-531, 2012.

6. Hakami A, Ali A and Hakami A: Effects of hepatitis B virus mutations on its replication and liver disease severity. Open Virol J 7: 12-18, 2013.

7. Jeong SW, Jang JY and Chung RT: Hepatitis C virus and hepatocarcinogenesis. Clin Mol Hepatol 18: 347-356, 2012.

8. Zhao Y, Jian W, Gao W, et al: RNAi silencing of c-Myc inhibits cell migration, invasion, and proliferation in HepG2 human hepatocellular carcinoma cell line: c-Myc silencing in hepatocellular carcinoma cell. Cancer Cell Int 13: 23-28, 2013.

9. Oster SK, Ho CS, Soucie EL and Penn LZ: The myc oncogene: MarvelouslY Complex. Adv Cancer Res 84: 81-154, 2002.

10. Pelengaris $\mathrm{S}$ and Khan M: The many faces of c-MYC. Arch Biochem Biophys 416: 129-136, 2003.

11. Facchini LM and Penn LZ: The molecular role of Myc in growth and transformation: recent discoveries lead to new insights. FASEB J 12: 633-651, 1998.

12. Schmidt EV: The role of $\mathrm{c}-\mathrm{myc}$ in cellular growth control. Oncogene 18: 2988-2996, 1999.

13. Boyd KE and Farnham PJ: Myc versus USF: discrimination at the cad gene is determined by core promoter elements. Mol Cell Biol 17: 2529-2537, 1997.

14. Bello-Fernandez C, Packham G and Cleveland JL: The ornithine decarboxylase gene is a transcriptional target of c-Myc. Proc Natl Acad Sci USA 90: 7804-7808, 1993.

15. Wu S, Pena A, Korcz A, Soprano DR and Soprano KJ: Overexpression of Mxi1 inhibits the induction of the human ornithine decarboxylase gene by the Myc/Max protein complex. Oncogene 12: 621-629, 1996.

16. Shim H, Dolde C, Lewis BC, Wu CS, Dang G, Jungmann RA, Dalla-Favera R and Dang CV: c-Myc transactivation of LDH-A: implications for tumor metabolism and growth. Proc Natl Acad Sci USA 94: 6658-6663, 1997.

17. Hubank M and Schatz DG: Identifying differences in mRNA expression by representational difference analysis of cDNA. Nucleic Acids Res 22: 5640-5648, 1994.

18. Perez-Roger I, Solomon DL, Sewing A and Land H: Myc activation of cyclin $\mathrm{E} / \mathrm{Cdk} 2$ kinase involves induction of cyclin $\mathrm{E}$ gene transcription and inhibition of p27(Kip1) binding to newly formed complexes. Oncogene 14: 2373-2381, 1997.

19. Galaktionov K, Chen X and Beach D: Cdc25 cell-cycle phosphatase as a target of c-myc. Nature 382: 511-517, 1996. 
20. Ou XM, Chen K and Shih JC: Monoamine oxidase A and repressor R1 are involved in apoptotic signaling pathway. Proc Natl Acad Sci USA 103: 10923-10928, 2006.

21. Huang A, Ho CS, Ponzielli R, et al: Identification of a novel c-Myc protein interactor, JPO2, with transforming activity in medulloblastoma cells. Cancer Res 65: 5607-5619, 2005.

22. Chen K, Ou XM, Chen G, et al: R1, a novel repressor of the human monoamine oxidase A. J Biol Chem 280: 11552-11559, 2005.

23. Johnson S, Stockmeier CA, Meyer JH, et al: The reduction of R1, a novel repressor protein for monoamine oxidase A, in major depressive disorder. Neuropsychopharmacology 36: 2139-2148, 2011.

24. Dai CX, Gao Q, Qiu SJ, et al: Hypoxia-inducible factor-1 alpha, in association with inflammation, angiogenesis and MYC, is a critical prognostic factor in patients with $\mathrm{HCC}$ after surgery. BMC Cancer 9: 418-428, 2009.

25. Dituri F, Mazzocca A, Peidro FJ, et al: Differential inhibition of the TGF-b signaling pathway in HCC cells using the small molecule inhibitor LY2157299 and the D10 monoclonal antibody against TGF-b receptor type II. PLoS One 8: 67109-67123, 2013.

26. Lin C-P, Liu C-R, Lee C-N, et al: Targeting c-Myc as a novel approach for hepatocellular carcinoma. World J Hepatol 2: 16-20, 2010

27. Prescott JE, Osthus RC, Lee LA, et al: A novel c-Myc-responsive gene, JPO1, participates in neoplastic transformation. J Biol Chem 276: 48276-48284, 2001.

28. Goto Y, Hayashi R, Muramatsu T, et al: JPO1/CDCA7, a novel transcription factor E2F1-induced protein, possesses intrinsic transcriptional regulator activity. Biochim Biophys Acta 1759: 60-68, 2006.
29. Osthus RC, Karim B, Prescott JE, et al: The Myc target gene JPO1/CDCA7 is frequently overexpressed in human tumors and has limited transforming activity in vivo. Cancer Res 65 : 5620-5627, 2005

30. Gill RM, Gabor TV, Couzens AL, et al: The MYC-associated protein CDCA7 is phosphorylated by AKT to regulate MYC-dependent apoptosis and transformation. Mol Cell Biol 33: 498-513, 2012.

31. Wang H, Wu K, Sun Y, et al: STC2 is upregulated in hepatocellular carcinoma and promotes cell proliferation and migration in vitro. BMB Rep 45: 629-634, 2012.

32. Sherr CJ: Cancer cell cycles. Science 274: 1672-1677, 1996.

33. Braun-Dullaeus RC, Mann MJ, Sedding DG, Sherwood SW, von der Leyen HE and Dzau VJ: Cell cycle-dependent regulation of smooth muscle cell activation. Arterioscler Thromb Vasc Biol 24: 845-850, 2004.

34. Braun-Dullaeus RC, Man MJ and Dzau VJ: Cell cycle progression: new therapeutic target for vascular proliferative disease. Circulation 98: 82-89, 1998

35. Tarn WY and Lai MC: Translational control of cyclins. Cell Div 6: 5-13, 2011.

36. Ajchenbaum F, Ando K, DeCaprio JA and Griffin JD: Independent regulation of human D-type cyclin gene expression during G1 phase in primary human T lymphocytes. J Biol Chem 268: 4113-4119, 1993.

37. Liu Y, Li W, Ye C, et al: Gambogic Acid induces G0/G1 cell cycle arrest and cell migration inhibition via suppressing PDGF receptor $\beta$ tyrosine phosphorylation and Racl activity in Rat aortic smooth muscle cells. J Atheroscler Thromb 17: 901-913, 2010. 